\title{
A Functional Representation of Fuzzy Preferences
}

\author{
Susheng Wang \\ Department of Economics, Hong Kong University of Science and Technology, Hong Kong, China \\ Email: s.wang@ust.hk
}

How to cite this paper: Wang, S.S. (2017) A Functional Representation of Fuzzy Preferences. Theoretical Economics Letters, 7, 13-22.

http://dx.doi.org/10.4236/tel.2017.71002

Received: October 26, 2016

Accepted: December 9, 2016

Published: December 13, 2016

Copyright $\odot 2017$ by author and Scientific Research Publishing Inc. This work is licensed under the Creative Commons Attribution International License (CC BY 4.0).

http://creativecommons.org/licenses/by/4.0/

\begin{abstract}
This paper defines a well-behaved fuzzy order and finds a simple functional representation for the fuzzy preferences. It includes the existing utility theory for exact preferences (no fuzziness) as a special case. It is a simple and intuitive extension of the utility theory under uncertainty, which can potentially be used to explain a few known paradoxes against the existing expected utility theory.
\end{abstract}

\section{Keywords}

Functional Representation, Utility Representation, Fuzzy Preferences

\section{Introduction}

A functional representation of individual preferences is a crucial issue in economics, especially when some puzzling experimental paradoxes against existing functional representations were found (such as the well-known Allais paradox and the St. Petersburg paradox). The non-expected utility approach is one way that many economists have tried in their effort to obtain a better functional representation of individual preferences ${ }^{1}$. This paper looks into the issue from a different angle: fuzzy preferences.

Traditional individual preferences are exact preferences, for which the preference of one consumption bundle over another is simply a yes or no. Fuzzy preferences, however, allow for a degree of preference of one consumption bundle over another. Exact preferences impose completeness in the sense that any two consumption bundles can be compared. Fuzzy preferences, on the other hand, allow for a continuum of attitudes towards preferences over any two choices. We may view fuzziness in preferences over consumption bundles as a version of weak completeness-that is, fuzziness measures the degree of completeness or vagueness.

Completeness of preferences, without any fuzziness, is used in the existing theory of utility representation and even in the theory of non-expected utility representation. However, many experimental investigations and real-life examples suggest that indi-

${ }^{1}$ See, for example, Kreps and Porteus [1], Epstein and Zin [2], and Weil [3]. 
vidual preferences do not necessarily satisfy the completeness axiom. In fact, some economists have argued that incomplete preferences may be a major cause of the problems found in the existing theories of utility representation of preferences ${ }^{2}$. One way to get around this problem is to replace completeness with fuzziness. As we will show, this generalization will still yield a simple functional representation. This new functional representation is much more general, but it still has many of the attractive properties of the existing theories of functional representation for exact preferences.

Fuzzy preferences have been extensively studied in the literature. Researchers in this area have found many interesting applications of fuzzy preferences to economic problem, especially to problems of rational choice and social choice, and, in particular, to the Orlovsky choice function and the Arrow impossibility theorem ${ }^{3}$. Our interest is in a functional representation of fuzzy preferences, which, to our knowledge, has never been done in the literature. With a convenient form of functional representation, theories based on fuzzy preferences become readily available for many economic problems, especially for those that employ function-based mathematical tools such as dynamic programming and functional analysis. This paper first finds a simple utility representation of fuzzy preferences, which includes the utility representation of exact preferences as a special case. It then finds a functional representation of fuzzy preferences, which also includes the functional representation of exact preferences as a special case.

This paper proceeds as follows. Section 2 provides three axioms that define a well-behaved fuzzy preference order. It includes the existing exact preference order as a special case. Some comparisons with existing definitions of fuzzy orders are discussed. Section 3 finds utility and functional representations of fuzzy preferences, which include existing functional representations of exact preferences as special cases. A particularly intuitive and convenient form of the functional representation is discussed (Theorem 3). Section 4 concludes the paper with a few remarks.

\section{Fuzzy Preferences}

Defining a proper class of fuzzy preferences is a major task in this paper. We do not intend to define a general class of fuzzy preferences that includes all possible fuzzy preferences. On the contrary, we will impose enough restrictions so as to obtain a neat functional representation of fuzzy preferences. Our intention is to strike a balance between generality and simplicity. We are interested in a class of representable fuzzy preferences.

Let $\mathbb{X} \subset \mathbb{R}^{n}$ be a closed set and $R: \mathbb{X}^{2} \rightarrow[-1,1]$ be a mapping. $R$ is a fuzzy order on $\mathbb{X}$ if it satisfies the following three axioms.

Axiom 1 (Reflexivity). $R(x, x)=0$, for all $x \in \mathbb{X}$.

Axiom 2 (Symmetry). $R(x, y)=-R(y, x)$, for all $x, y \in \mathbb{X}$.

Axiom 3 (Transitivity). For any $x, y \in \mathbb{X}$, if there exists $z \in \mathbb{X}$ such that $R(x, z) \geq 0$ and $R(z, y) \geq 0$, then $R(x, y) \geq 0$.

A fuzzy order $R$ is an exact order if its range $R\left(\mathbb{X}^{2}\right)$ contains only three values

${ }^{2}$ See, for example, Schmeidler [4], Flament [5], and Gay [6].

${ }^{3}$ See, for example, Basu [7], Dutta [8], Barrett et al. [9], Banerjee [10], Dasguptaand Deb [11], Richardson [12], and Sengupta [13] and [14]. 
$-1,0$ and 1 , i.e., $R\left(\mathbb{X}^{2}\right)=\{-1,0,1\}$. That is, an exact order is a special fuzzy order. For an exact order, the three cases $R(x, y)=-1,0$ and 1 are respectively interpreted as strictly less preferred, indifferent, and strictly preferred.

Exact orders describe traditional preferences, which are reflexive, transitive, and complete. A fuzzy order is, in certain sense, an extension of an exact order to an order with weak completeness. In other words, the preference of a consumption bundle over another is no longer a simple yes/no, rather, it has a degree of preference.

As we know, under certain conditions, traditional preferences have utility and functional representations. The task of this paper is to show that the existing utility and functional representations of exact preferences can be extended to fuzzy preferences. In other words, we will find conditions under which fuzzy preferences have utility and functional representations, and we will show that the existing utility and functional representations of exact preferences are special cases.

In order to achieve a neat functional representation of fuzzy preferences, we must impose restrictions over the standard notion of fuzzy preferences found in the literature. Our axioms impose sufficient restrictions on fuzzy preferences and at the same time allow enough generality to cover a large class of sensible preferences.

The symmetry axiom above is the key restriction. Specifically, the existing definition of a fuzzy order in the literature is a mapping $\bar{R}: \mathbb{X}^{2} \rightarrow[0,1]$, as opposed to our definition of a mapping $R: \mathbb{X}^{2} \rightarrow[-1,1]$. Although we can convert the two definitions literally by the formula $\bar{R}(x, y)=[1+R(x, y)] / 2$, the interpretations of their values are different. As opposed to interpreting $\bar{R}(x, y)$ as the degree to which $x$ is better than $y$, we interpret $|R(x, y)|$ as the degree to which $x$ is better than $y$ and $-|R(x, y)|$ as the degree to which $x$ is worse than $y$. Thus, according to our interpretation, we should have $R(x, y)=-R(y, x)$. This is the symmetry axiom. Technically, our symmetry axiom is a special case of the standard completeness axiom of $\bar{R}(x, y)+\bar{R}(y, x) \geq 1$ or $R(x, y) \geq-R(y, x)$ in the literature.

With the symmetry axiom, we can intuitively define the concept of indifference. If $R(x, y)=0$, we say that $x$ and $y$ are indifferent and denote their relation as $x \sim y^{4}$. The reflexivity axiom requires a consumption bundle to be indifferent to itself: $x \sim x$.

Our transitivity axiom is new. There are quite a few alternative formulations of transitivity in the existing literature, among which the following three are popular:

a) $R(x, y)>R(x, z)+R(z, y)-1, \forall x, y, z \in \mathbb{X}$.

b) $R(x, y) \geq \frac{1}{2} R(x, z)+\frac{1}{2} R(z, y), \quad \forall x, y, z \in \mathbb{X}$.

c) $R(x, y) \geq \min \{R(x, z), R(z, y)\}, \quad \forall x, y, z \in \mathbb{X}$.

Each of these formulations has its problem. For (a), the problem can be explained by an example. If $x \sim z$ and $z \sim y$, we should expect $x \sim y$. But condition (a) only suggests $R(x, y)>-1$, i.e., $x \nprec y$.

For condition (b), consider $R(x, z)=-1$ and $R(z, y)=1$. We should not expect these two conditions to tell us anything about the relation between $x$ and $y$. However, condition (b) implies $R(x, y) \geq 0$.

${ }^{4}$ Similarly, we can denote $x \succ y$ for $R(x, y)=1$, and $x \prec y$ for $R(x, y)=-1$. 
For condition (c), as pointed out by Basu [7] (footnote 3), consider $R(x, z)=0, R\left(x^{\prime}, z\right)=1$ and $R(z, y)=0$. Since condition (c) indicates $R(x, y) \geq 0$, we should expect the lower bound on $R\left(x^{\prime}, y\right)$ to be strictly larger than 0 , but condition (c) continues to indicate $R\left(x^{\prime}, y\right) \geq 0$.

Our version of transitivity is stated in a way that appears to be a natural extension of the transitivity axiom for exact preferences. Because of the symmetry axiom, we do not need an elaborate formula to define our version of transitivity, and our notion of transitivity appears to be less restrictive than other existing notions of transitivity. In particular, for our notation of transitivity, the sizes of $R(x, z)$ and $R(z, y)$ have nothing to do with the size of $R(x, y)$ This avoids the above mentioned problems. In addition, our transitivity axiom implies the usual transitivity axiom for exact orders as a special case.

Lemma 1. For any fuzzy order $R(\cdot, \cdot)$, if, for some $z \in \mathbb{X}, R(x, z) \geq 0$ and $R(z, y) \geq 0$ and one of them is strict, then $R(x, y)>0$.

Proof: Suppose not. Suppose $R(x, z)>0$ and $R(z, y) \geq 0$ but $R(x, y)=0$. Then, we have $R(z, y) \geq 0$ and $R(y, x)=0$. By transitivity, we then have $R(z, x) \geq 0$ or $R(x, z) \leq 0$. This contradicts with the fact $R(x, z)>0$. Therefore, we must have $R(x, y)>0$.

Lemma 1 suggests that the usual transitivity condition for exact orders is a special case of our transitivity condition for fuzzy orders.

Lemma 2. If $x \sim z$ and $z \sim y$, then $x \sim y$.

Proof: By transitivity, $R(x, z)=0$ and $R(z, y)=0$ implies $R(x, y) \geq 0$. By symmetry, we also have $R(y, z)=0$ and $R(z, x)=0$, which then implies $R(y, x) \geq 0$, i.e., $R(x, y) \leq 0$. Thus, $R(x, y)=0$

Lemma 2 . is a standard property of preferences. This property is essential for a functional representation.

\section{Functional Representation}

We say that a function $u: \mathbb{X} \rightarrow \mathbb{R}$ represents fuzzy preferences $R: \mathbb{X}^{2} \rightarrow[-1,1]$ if, for all $x, y \in \mathbb{X}, u(x)>u(y) \Leftrightarrow R(x, y)>0$ and $u(x)=u(y) \Leftrightarrow x \sim y$.

It turns out that the utility representation theorem for fuzzy preferences is similar to the one for exact preferences. It has similar axioms and similar proofs. In fact, utility and functional representations of exact preferences are special cases of the ones for fuzzy preferences.

For an exact order $R: \mathbb{X}^{2} \rightarrow\{-1,0,1\}$, under continuity, we know that there exists a continuous function $u: \mathbb{X} \rightarrow \mathbb{R}$ and a monotone function $\varphi: \mathbb{R}^{2} \rightarrow \mathbb{R}$ such that

$$
R(x, y)=\varphi[u(x), u(y)], \forall x, y \in \mathbb{X}
$$

In fact, this function $\varphi$ is the sign function $\varphi(a, b)=\operatorname{sign}(a-b)$ where the sign function sign : $\mathbb{R} \rightarrow\{-1,0,1\}$ is defined by

$$
\operatorname{sign}(t)= \begin{cases}-1, & \text { if } t<0 \\ 0, & \text { if } t=0 \\ 1, & \text { if } t>0\end{cases}
$$


For a fuzzy order $R: \mathbb{X}^{2} \rightarrow[-1,1]$, under what conditions, can we find a continuous function $u: \mathbb{X} \rightarrow \mathbb{R}$ and a monotone function $\varphi: \mathbb{R} \rightarrow[-1,1]$ such that (1) holds? We call $u$ a utility representation and $(u, \varphi)$ a functional representation. If (1) is true for fuzzy orders, it means that the utility representation and functional representations for exact preferences are special cases.

\subsection{Abbreviations and Acronyms}

To establish (1), just as for exact preferences, we need the following two axioms.

Axiom 4 (Continuity). $\left\{x \in \mathbb{X} \mid R\left(x, x_{0}\right) \geq 0\right\}$ and $\left\{t \in \mathbb{X} \mid R\left(x, x_{0}\right) \leq 0\right\}$ are closed in $\mathbb{X}, \forall x_{0} \in \mathbb{X}$.

Axiom 5 (Monotonicity). $x \geq y \Rightarrow R(x, y) \geq 0$.

We also need the following two lemmas for the proof of the utility representation theorem.

Lemma 3. Any $A \subset \mathbb{R}$ is connected $\Leftrightarrow A$ is an interval.

Lemma 4. A function $f: \mathbb{R}_{+}^{k} \rightarrow \mathbb{R}$ is continuous $\Leftrightarrow f^{-1}(a, b)$ is open in $\mathbb{R}_{+}^{k}, \forall a, b \in \mathbb{R}$.

Theorem 1 (Utility Representation). There is a continuous utility representation $u: \mathbb{X} \rightarrow \mathbb{R}$ for any continuous fuzzy preferences $R: \mathbb{X}^{2} \rightarrow[-1,1]$.

Proof: This proof is similar to the proof of the same result for exact orders. For simplicity of the proof, we assume strictly monotonic preferences, that is, in addition to Axiom 5 , we further require $x>y \Rightarrow R(x, y)>0$. Also, let $\mathbb{X} \subset \mathbb{R}^{n}$ and $e \equiv(1,1, \cdots, 1) \in \mathbb{X}$. For any $x \in \mathbb{X}$, we need to find a unique number $u(x)$ such that $x \sim u(x) e$. Given $x \in \mathbb{X}$, define

$$
A \equiv\left\{t \in \mathbb{R}_{+} \mid R(t e, x) \geq 0\right\}, B \equiv\left\{t \in \mathbb{R}_{+} \mid R(t e, x) \leq 0\right\} .
$$

It is obvious that $A \cup B=\mathbb{R}_{+}$. By continuity, $A$ and $B$ are closed sets in $\mathbb{R}_{+}$. By monotonicity, $A \neq \varnothing$ and $B \neq \varnothing$. If $A \cap B=\varnothing$, then $A^{c} \equiv \mathbb{R}_{+} \backslash A=B$ and $B^{c} \equiv \mathbb{R}_{+} \backslash B=A$, implying that $A$ and $B$ are open sets in $\mathbb{R}_{+}$. Since $\mathbb{R}_{+}$is connected by Lemma $3, A$ or $B$ must be empty. This is a contradiction. Therefore, $A \cap B \neq \varnothing$.

Suppose $t_{1}, t_{2} \in A \cap B$. Then $R\left(t_{1} e, x\right)=R\left(t_{2} e, x\right)=0$, implying $t_{1} e \sim t_{2} e$. By strict monotonicity, $t_{1}=t_{2}$. Therefore, $A \cap B$ only contains a single point. Let $u(x)$ denote this point. Then, $x \sim u(x) e$ (Figure 1 ).

We now show that this function $u(x)$ represents the preferences. By the definition of $u(x)$ and strong monotonicity,

$$
u(x)>u(y) \Leftrightarrow u(x) e>u(y) e \Leftrightarrow R[u(x) e, u(y) e]>0 \Leftrightarrow R(x, y)>0,
$$

and

$$
u(x)=u(y) \Leftrightarrow x \sim u(x) e=u(y) e \sim y \Leftrightarrow x \sim y
$$

$u$ thus represents the preferences.

We now only need to show the continuity. For any $a, b \in \mathbb{R}$, we have $\mathrm{e}^{5}$

${ }^{5}$ For any sets $A, B$, and function $f$, we have $f^{-1}\left(A^{c}\right)=\left[f^{-1}(A)\right]^{c}$ and $f^{-1}(A \cup B)=f^{-1}(A) \cup f^{-1}(B)$. 


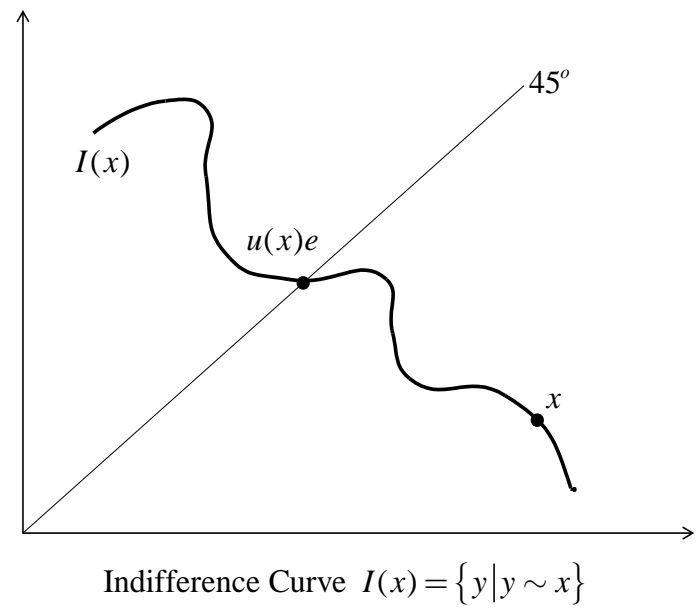

Figure 1. Utility representation.

$$
\begin{aligned}
u^{-1}(a, b) & =u^{-1}\left[\{(-\infty, a] \cup[b, \infty)\}^{c}\right]=\left\{u^{-1}[(-\infty, a] \cup[b, \infty)]\right\}^{c} \\
& =\left\{u^{-1}(-\infty, a] \cup u^{-1}[b, \infty)\right\}^{c}=\left\{u^{-1}(-\infty, a]\right\}^{c} \cap\left\{u^{-1}[b, \infty)\right\}^{c} \\
& =[\{x \in X \mid u(x) \leq a\}]^{c} \cap[\{x \in X \mid u(x) \geq b\}]^{c} .
\end{aligned}
$$

Since $u(x) \leq a \Leftrightarrow R[u(x) e, a e] \leq 0 \Leftrightarrow R(x, a e) \leq 0$, we then have

$$
u^{-1}(a, b)=[\{x \in \mathbb{X} \mid R(x, a e) \leq 0\}]^{c} \cap[\{x \in \mathbb{X} \mid R(x, b e) \geq 0\}]^{c} .
$$

By continuity, $u^{-1}(a, b)$ is thus open. Hence, by Lemma $4, u(x)$ is continuous.

As we know, the continuity condition is necessary for the existence of a utility representation for exact preferences. It must therefore be necessary for fuzzy preferences. The transitivity condition is sufficient for Lemma 1. Since Theorem 1 implies Lemma 1, the transitivity condition must also be necessary for the existence of a utility representation for fuzzy preferences.

Remark 1 . The utility representation of fuzzy preferences appears to be the same as the traditional utility representation of exact preferences. However, the functional representation of fuzzy preferences in the following two sections will show the crucial differences. In fact, the interesting part of a representation theory for fuzzy preferences is its functional representation, from which the key differences between fuzzy and exact preferences are revealed clearly.

\subsection{Functional Representation}

We now proceed to find a functional representation for fuzzy preferences.

Lemma 5. For exact preferences $R: \mathbb{X}^{2} \rightarrow\{-1,0,1\}, x \sim x^{\prime}$ and $y \sim y^{\prime}$ imply $R(x, y)=R\left(x^{\prime}, y^{\prime}\right)$.

Proof: If $R(x, y)=0$, i.e. $x \sim y$, then, by Lemma 2, $x^{\prime} \sim y$. By Lemma 2 again, using $x^{\prime} \sim y$ and $y \sim y^{\prime}$, we have $x^{\prime} \sim y^{\prime}$. Thus, $R(x, y)=R\left(x^{\prime}, y^{\prime}\right)$. 
If $R(x, y)=-1$, i.e. $x \prec y$, then, by Lemma $1, x^{\prime} \prec y$. By Lemma 1 again, using $x^{\prime} \prec y$ and $y \sim y^{\prime}$, we have $x^{\prime} \prec y^{\prime}$, i.e., $R\left(x^{\prime}, y^{\prime}\right)=-1$. Thus, $R(x, y)=R\left(x^{\prime}, y^{\prime}\right)$.

Similarly, for $R(x, y)=1$, we also have $R(x, y)=R\left(x^{\prime}, y^{\prime}\right)$. The lemma is thus proved.

For fuzzy preferences, we will impose Lemma 5 as an axiom, which is needed for a functional representation.

Axiom 6 (Independence). $x \sim x^{\prime}$ and $y \sim y^{\prime}$ imply $R(x, y)=R\left(x^{\prime}, y^{\prime}\right)$.

The independence axiom states that the degree of preference stays the same after an indifference transformation. In the expected utility literature, this axiom is known as the independence axiom, which is crucial for the existence of an expected utility representation of preferences under uncertainty. For our fuzzy preferences, this axiom is crucial for the existence of a functional representation of preferences under fuzziness.

The following lemma is from Protter and Morrey [15].

Lemma 6 (Tietze Extension Theorem). Let $A$ be a closed set in a metric space $S$ and $f: A \rightarrow \mathbb{R}$ a continuous and bounded function. Define $M=\sup _{x \in A}|f(x)|$. Then there is a continuous function $g: S \rightarrow \mathbb{R}$ such that

$$
g(x)=f(x), x \in A ; \text { and } g(x) \leq M, x \in S .
$$

Theorem 2 (Functional Representation).Given a continuous fuzzy order $R: \mathbb{X}^{2} \rightarrow[-1,1]$ and its utility representation $u: \mathbb{X} \rightarrow \mathbb{R}$ let $\mathbb{U} \equiv u(\mathbb{X})$ Then, the fuzzy order is independent if and only if there exists a function $\varphi: \mathbb{U}^{2} \rightarrow[-1,1]$ such that $\varphi[u(x), u(y)]=R(x, y)$ for all $x, y \in \mathbb{X}$, and

a) $\varphi\left(t_{1}, t_{2}\right)=-\varphi\left(t_{2}, t_{1}\right), \forall t_{1}, t_{2} \in \mathbb{U}$,

b) $\varphi(t, t)=0, \forall t \in \mathbb{U}$,

c) $\varphi\left(t_{1}, t_{2}\right) \geq 0$ and $\varphi\left(t_{2}, t_{3}\right) \geq 0 \Rightarrow \varphi\left(t_{1}, t_{3}\right) \geq 0$.

Conversely, given a continuous function $u: \mathbb{X} \rightarrow \mathbb{R}$ and a function $\varphi: \mathbb{U}^{2} \rightarrow[-1,1]$ satisfying the above three conditions, define a function $R: \mathbb{X}^{2} \rightarrow[-1,1]$ by $R(x, y) \equiv \varphi[u(x), u(y)]$. Then, this $R$ is a fuzzy order. Furthermore, if $\mathbb{X}$ is compact and $R$ is continuous, $\varphi$ is also continuous and can be defined on $\mathbb{R}^{2}$.

Proof: Independence is obviously necessary. We only prove its sufficiency.

By Theorem 1, there exists a continuous utility representation $u: \mathbb{X} \rightarrow \mathbb{R}$. By independence, we can simply define $\varphi: \mathbb{U}^{2} \rightarrow[-1,1]$ such that, for $\left(u_{1}, u_{2}\right) \in \mathbb{U}^{2}$,

$$
\varphi\left(u_{1}, u_{2}\right)=R(x, y) \text {, where } u_{1}=u(x), u_{2}=u(y) \text {. }
$$

By independence, given any $\left(u_{1}, u_{2}\right) \in \mathbb{U}^{2}$, we have

$$
R(x, y)=R\left(x^{\prime}, y^{\prime}\right) \text {, for any other }\left(x^{\prime}, y^{\prime}\right) s \cdot t \cdot u_{1}=u\left(x^{\prime}\right), u_{2}=u\left(y^{\prime}\right) .
$$

The function $\varphi$ is thus well defined.

By symmetry $R(x, y)=-R(y, x)$, we immediately have $\varphi\left(t_{1}, t_{2}\right)=-\varphi\left(t_{2}, t_{1}\right)$, for all $t_{1}, t_{2} \in u(\mathbb{X})$. By reflexivity, we have $\varphi(t, t)=0, \forall t \in u(\mathbb{X})$. The transitivity implies

$$
\varphi\left(t_{1}, t_{2}\right) \geq 0 \text { and } \varphi\left(t_{2}, t_{3}\right) \geq 0 \Rightarrow \varphi\left(t_{1}, t_{3}\right) \geq 0 \text {. }
$$

If $\mathbb{X}$ is compact and $R$ is continuous, then $\mathbb{U}$ is closed and $\varphi$ is also continuous. By the Tietze Extension Theorem, we can extend $\varphi$ to be a continuous function on $\mathbb{R}^{2}$. 
The converse is straightforward.

The three conditions (a), (b) and (c) in Theorem 2 are necessary for a function $\varphi: \mathbb{U} \rightarrow[-1,1]$ that defines a fuzzy order.

A good example of Theorem 2 is the exact preferences with $\varphi(a, b) \equiv \operatorname{sign}(a-b)$. For an exact preference order $R: \mathbb{X}^{2} \rightarrow\{-1,0,1\}$, the functional representation is

$$
R(x, y)=\operatorname{sign}[u(x)-u(y)], \forall x, y \in \mathbb{X} .
$$

This is a special case of Theorem 2 , in which $\varphi\left(t_{1}, t_{2}\right) \equiv \operatorname{sign}\left(t_{1}-t_{2}\right)$. In this case, we obviously have $\varphi\left(t_{1}, t_{2}\right)=-\varphi\left(t_{2}, t_{1}\right)$ and $\varphi(t, t)=0, \forall t_{1}, t_{2}, t \in \mathbb{R}$.

\subsection{A Special Form of the Functional Representation}

We now proceed to find a more convenient form of the functional representation.

Theorem 3. Given a utility representation $u: \mathbb{X} \rightarrow \mathbb{R}$ of a fuzzy order $R: \mathbb{X}^{2} \rightarrow[-1,1]$, if and only if

$$
u(x)-u(y)=u\left(x^{\prime}\right)-u\left(y^{\prime}\right) \text { implies } R(x, y)=R\left(x^{\prime}, y^{\prime}\right),
$$

there exists $\varphi: \mathbb{R} \rightarrow[-1,1]$ such that

$$
R(x, y)=\varphi[u(x)-u(y)] \text {, for all } x, y \in \mathbb{X} .
$$

Proof: The proof is straightforward-what we need to do is to define the function $\varphi$ directly. We define the function $\varphi: \mathbb{R} \rightarrow[-1,1]$ by

$$
\varphi[u(x)-u(y)]=R(x, y), \text { for } x, y \in \mathbb{X} .
$$

By condition (2), this function is well defined.

Formula (3) is particularly convenient to use. It is also very close to the utility representation of exact preferences. The interesting part of the representation (3) is that we can treat the difference of utility values $u(x)-u(y)$ as the preference intensity difference between two consumption bundles $x$ and $y$, and the role of $\varphi$ here is to convert a value $t \in \mathbb{R}$ to a value $\varphi(t) \in[-1,1]$ so that we can call it a degree of preferences.

With Theorem 3, we can choose special forms of $\varphi$ to define interesting fuzzy orders $R$. For example, for $u: \mathbb{X} \rightarrow[0,1]$, define

$$
R(x, y)=u(x)-u(y) .
$$

Here, implicitly $\varphi\left(t_{1}, t_{2}\right) \equiv t_{1}-t_{2}$. Since this $\varphi$ satisfies the three conditions (a), (b) and (c) in Theorem 2, the fuzzy preferences in (4) is hence well defined.

We can also define

$$
R(x, y)=\operatorname{sign}[u(x)-u(y)] .
$$

Here, implicitly $\varphi\left(t_{1}, t_{2}\right) \equiv \operatorname{sign}\left(t_{1}-t_{2}\right)$. This $\varphi$ also satisfies the three conditions (a), (b) and (c) in Theorem 2. The fuzzy preferences in Banerjee is hence also well defined. In fact, this preference order describes the traditional exact preferences.

Remark 2. We can now understand Remark 1 intuitively. The difference between fuzzy and exact preferences is not in the direction of preferences but in the strength of preferences, and such strength may be distributed disproportionally across the utility space $\mathbb{U}$. This explains the similarity of utility representations for fuzzy and exact preferences. The utility function $u$ indicates the direction of preferences, and the func- 
tional $\varphi$ represents the distribution of strength of preferences. In special cases, the strength can be distributed proportionally as in (4), and it can also be distributed in a yes/no fashion as in Banerjee.

\section{Concluding Remarks}

What this paper has accomplished are: first, to define a well-behaved fuzzy order; and second, to find a simple functional representation for the fuzzy preferences. It is a general utility and functional representation theory, which includes the existing utility and functional representation theory for exact preferences as a special case. In doing so, we have tried to obtain a theory that is simple and intuitive.

Such a theory may be readily incorporated into a utility theory under uncertainty. A more general/flexible utility theory has the potential to explain a few well-known paradoxes, such as the Allais paradox and St. Petersburg paradox. The advantage of this approach is that it is still within the framework of expected utility, as opposed to non-expected utility.

\section{References}

[1] Kreps, D. and Porteus, E (1978) Temporal Resolution of Uncertainty and Dynamic Choice Theory. Econometrica, 97, 91-100. https://doi.org/10.2307/1913656

[2] Epstein, L.G. and Zin, S.E. (1989) Substitution, Risk Aversion and the Temporal Behavior of Consumption and Asset Returns: A Theoretical Framework. Econometrica, 57, 937-969. https://doi.org/10.2307/1913778

[3] Weil, P. (1990) Nonexpected Utility in Macroeconomics. Quarterly Journal of Economics, 105, 29-42. https://doi.org/10.2307/2937817

[4] Schmeidler, D. (1969) Competitive Equilibria in Markets with a Continuum of Traders and Incomplete Preferences. Econometrica, 37, 578-585. https://doi.org/10.2307/1910435

[5] Flament, C. (1983) On Incomplete Preference Structures.Mathematical Social Sciences, 5, 61-72. https://doi.org/10.1016/0165-4896(83)90085-9

[6] Gay, A. (1992) Complete vs. Incomplete Preferences and Economic Behavior. In Pasinetti, L., Ed., Italian Economic Papers, Volume 1, 123-188, Oxford University Press, Oxford, UK.

[7] Basu, K. (1984) Fuzzy Revealed Preference Theory. Journal of Economic Theory, 32, 212-227. https://doi.org/10.1016/0022-0531(84)90051-6

[8] Dutta, B. (1987) Fuzzy Preferences and Social Choice. Mathematical Social Sciences, 13, 215-29. https://doi.org/10.1016/0165-4896(87)90030-8

[9] Barrett, C.R., Pattanaik, P.K. and Salles, M. (1990) On Choosing Rationally When Preferences Are Fuzzy. Fuzzy Sets and Systems, 34, 197-212. https://doi.org/10.1016/0165-0114(90)90159-4

[10] Banerjee, A. (1993) Rational Choice under Fuzzy Preferences: The Orlovsky Choice Function. Fuzzy Sets and Systems, 53, 295-299. https://doi.org/10.1016/0165-0114(93)90375-R

[11] Dasgupta, M. and Deb, R. (1996) Transitivity and Fuzzy Preferences. Social Choice and Welfare, 13, 305-318. https://doi.org/10.1007/BF00179234

[12] Richardson, G. (1998) The Structure of Fuzzy Preferences: Social Choice Implications. Social Choice and Welfare, 15, 359-369. https://doi.org/10.1007/s003550050111

[13] Sengupta, K. (1998) Fuzzy Preference and Orlovsky Choice Procedure. Fuzzy Sets and Systems, 93, 231-234. https://doi.org/10.1016/S0165-0114(96)00079-6

[14] Sengupta, K. (1999) Choice Rules with Fuzzy Preferences: Some Characterizations. Social 
Choice and Welfare, 16, 259-272. https://doi.org/10.1007/s003550050143

[15] Protter, M.H. and Morrey, C.B. (1991) A First Course in Real Analysis. Springer-Verlag, New York. https://doi.org/10.1007/978-1-4419-8744-0

Submit or recommend next manuscript to SCIRP and we will provide best service for you:

Accepting pre-submission inquiries through Email, Facebook, LinkedIn, Twitter, etc. A wide selection of journals (inclusive of 9 subjects, more than 200 journals)

Providing 24-hour high-quality service

User-friendly online submission system

Fair and swift peer-review system

Efficient typesetting and proofreading procedure

Display of the result of downloads and visits, as well as the number of cited articles

Maximum dissemination of your research work

Submit your manuscript at: http://papersubmission.scirp.org/

Or contact tel@scirp.org 